\title{
Renal vein injury complicating removal of intravenous leiomyoma
}

\author{
Farzan Filsoufi, MD, R. Saeid Farivar, MD, PhD, Curtis Anderson, MD, Dean Santerre, and David H. Adams, MD, \\ Boston, Mass
}

I ntravenous leiomyoma is a benign tumor with an extremely low incidence, first described by Knauer ${ }^{1}$ in 1902 , with at least 33 reports involving the right ventricle. The tumor extends from the uterine/ovarian vein to the iliac vein and inferior vena cava (IVC), eventually reaching the right side of the heart. The tumor has signs and symptoms of IVC occlusion and the typical sequelae of right heart failure, ${ }^{2}$ including hepatomegaly, ascites, peripheral edema, and dyspnea. Sudden cardiac death due to the total obstruction of the tricuspid valve ${ }^{3}$ has also been reported. Herein we report an avulsion injury of the renal vein

From the Division of Cardiac Surgery, Brigham and Women's Hospital, Harvard Medical School, Boston, Mass.

Received for publication June 26, 2001; accepted for publication July 27, 2001.

Address for reprints: David H. Adams, MD, Department of Cardiothoracic Surgery, Mount Sinai Medical Center, 1190 Fifth Ave, Suite 1028, New York, NY 10029 (E-mail: david.adams@mountsinai.org).

J Thorac Cardiovasc Surg 2002;123:820-2

Copyright (C) 2002 by The American Association for Thoracic Surgery

$0022-5223 / 2002 \$ 35.00+0 \quad \mathbf{1 2 / 5 4 / 1 2 0 3 2 6}$

doi:10.1067/mtc.2002.120326 during extraction of a right atrial tumor without abdominal exposure. We believe our experience suggests that a 1-stage approach with venous exposure at the time of tumor extraction would be safer in future cases.

\section{Clinical Summary}

A 40-year-old woman presented for evaluation of progressive dyspnea. The physical examination was significant for morbid obesity and peripheral edema, with a holosystolic murmur (III/VI) at the right sternal border. Results of the examination were otherwise unremarkable. The diagnostic workup included a transthoracic and transesophageal echocardiogram, which revealed a mass in the right atrium and ventricle, which had an echogenicity that favored a tumor (versus thrombus; Figure 1, A). By echocardiography the mass was equal in size to the tricuspid orifice and was obstructing flow. A magnetic resonance imaging study demonstrated a large mass in the pelvis and an intraluminal mass in the IVC (Figure 1, $B$ ), extending to the right side of the heart and the left renal vein. It was noted by magnetic resonance imaging that the IVC and pelvic veins below the left renal vein were patent with no intraluminal masses. The patient was scheduled for a 2-stage operation-removal of the tumor from the circulation to be followed by completion oophorectomy. 

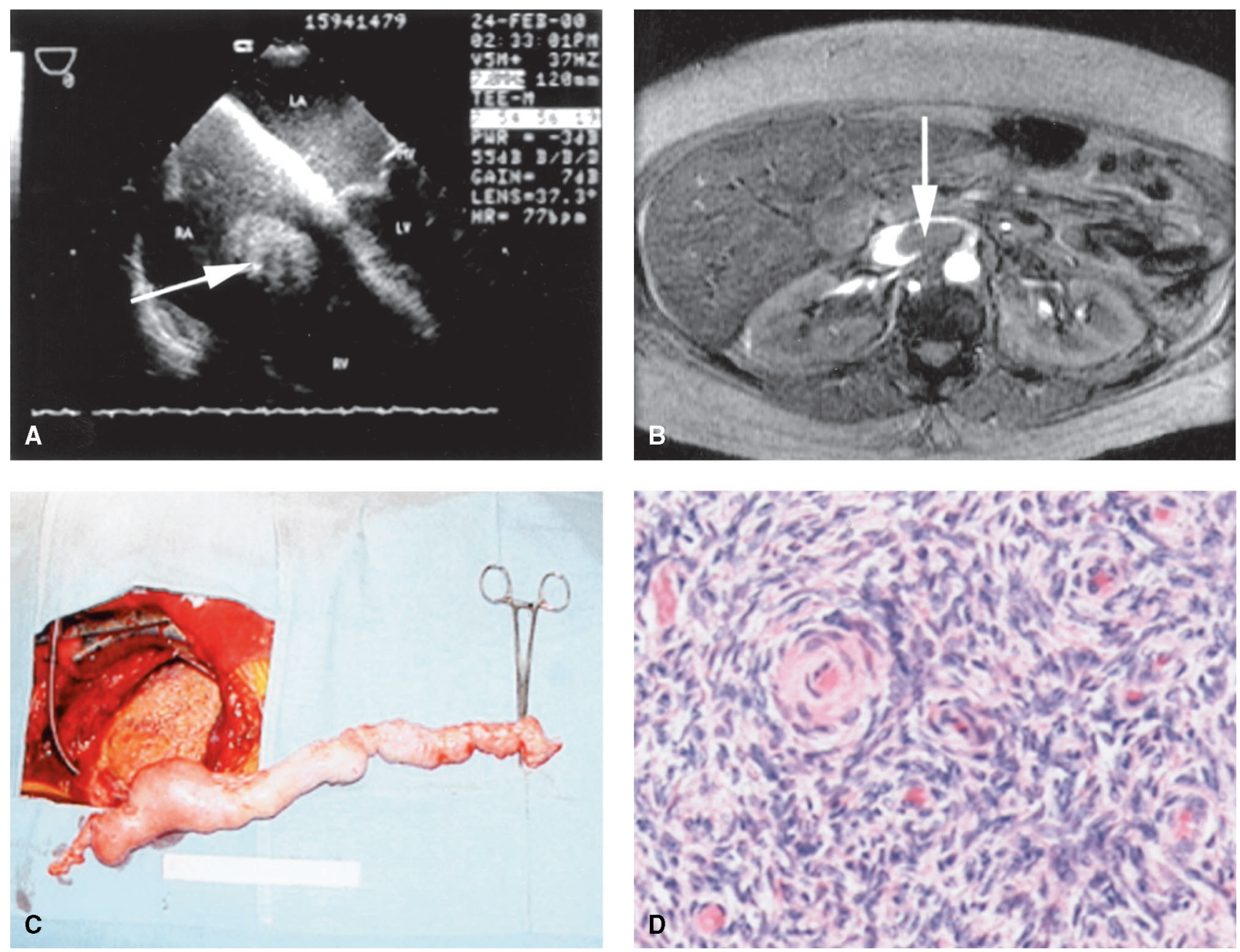

Figure 1. A, Echocardiogram demonstrating a mass (arrow) through the tricuspid valve. B, Axial magnetic resonance imaging study demonstrating a mass (arrow) in the lumen of the IVC. C, Extensive leiomyoma at operation with a clamp on the distal tumor. D, Hematoxylin and eosin staining of the leiomyoma, with characteristic smooth muscle cells (original magnification $400 \times$ ). $L A$, Left atrium; $L V$, left ventricle; $R A$, right atrium; $R V$, right ventricle.

The patient underwent surgery with ascending aortic, superior vena caval, and left femoral venous cannulation and institution of cardiopulmonary bypass with assisted venous drainage. Hypothermia was instituted to $20^{\circ} \mathrm{C}$ to allow reduced blood flow to 500 to $1000 \mathrm{~mL} / \mathrm{min}$ during tumor extraction. A right atriotomy was performed, and a massive tumor was found extending from the right atrium through the tricuspid valve into the right ventricle. The intracardiac mass was removed en bloc after gentle traction was applied to the tumor limb extending from the IVC. The tumor limb was easily mobilized from the IVC wall (Figure 1, $C$ ), and histologic examination confirmed the diagnosis of leiomyoma (Figure $1, D)$. The right atrium was closed and the patient was rewarmed. During this time the patient had a significant volume requirement, presumably because of vasodilation, which at first responded to pharmacologic vasoconstriction. The perfusionist brought to our attention a persistent requirement for added volume to keep the cardiotomy reservoir adequately filled. Because of the patient's obesity, we could not appreciate any change in her abdominal girth. Nonetheless, we elected to perform an exploratory laparotomy to rule out intra-abdominal venous catastrophe.

During exploration, a large hematoma was noted in the left pelvis at the margin of the uterus. The left ovarian vein had been avulsed from the left renal vein, presumably as a result of traction on the leiomyoma on the renal vein. Patch angioplasty of the left renal vein was performed with autologous pericardium. Bilateral oophorectomy was performed and hysterectomy was deferred until 3 months later. The patient is currently well after 1 year of follow-up. A postoperative echocardiogram demonstrated a normal-sized right ventricle and right atrium with trivial tricuspid regurgitation.

\section{Discussion}

Intravenous leiomyoma with extension to the cardiac structures is a rare diagnosis. Recurrence has been noted unless a radical 
resection, including hysterectomy, is performed. However, even with complete hysterectomy, recurrence may occur. The precise cause is unknown. There is controversy as to the origin of the smooth muscle cells, because some advocate an origin in the smooth muscle cells of the uterine wall, whereas others claim an origin in the media of the uterine or ovarian vein. It is unknown whether the leiomyoma represents a monoclonal or polyclonal proliferation of cells. Although the tumor is benign, its intraluminal location can lead to symptoms of right heart failure and tricuspid valve obstruction, which may lead to sudden cardiac death. $^{3}$

Various surgical approaches have been used, including a 1 -stage radical resection or a 2-stage resection. One-stage resections have been reported on bypass with normothermia ${ }^{4}$ or with hypothermic circulatory arrest. ${ }^{5}$ Two-stage procedures have been advocated for extensive leiomyomatosis. ${ }^{2}$

In this patient, the leiomyoma extended into the left ovarian and left renal veins. Originally, we had opted for a 2-stage procedure. Despite the intraoperative impression that the tumor was not adherent to the wall of the IVC, a traction injury of the left renal vein necessitated an urgent laparotomy. The venous injury was repaired satisfactorily with autologous pericardium. To our knowledge, this is the first reported avulsion injury of the renal and ovarian veins on removal of an intravenous leiomyoma. Persistent volume loss after tumor removal in a double-stage approach should prompt an urgent laparotomy to rule out significant venous injury. On the basis of our experience with this case, we now recommend a single-stage approach with abdominal venous exposure at the time of tumor extraction.

\section{References}

1. Knauer E. Zur Anatomic der Uterusmyome; 1902.

2. Casillas J, Lobato J, Llamas P, Meseguer J. Intracardiac extension of intravenous leiomyomatosis. J Cardiovasc Surg. 1997;38:83-6.

3. Bahary C, Gorodeski I, Nilly M, Neri A, Avidor I, Garti IJ. Intravascular leiomyomatosis. Obstet Gynecol. 1982;59:73s-7s.

4. Wakiyama H, Sugimoto T, Ataka K, Yamashita C, Tsuji Y, Nakagiri $\mathrm{K}$, et al. Intravenous leiomyomatosis extending into the right ventricular cavity: one-stage radical operation using cardiopulmonary bypass. Angiology. 2000;51:505-9.

5. Steinmetz O, Bedard P, Prefontaine M, Bourke M, Barber G. Uterine tumor in the heart: intravenous leiomyomatosis. Surgery. 1995;119: 226-9. 